$5-1-2015$

\title{
SPSS Programs for Addressing Two Forms of Power for Multiple Regression Coefficients
}

Christopher Aberson

Humboldt State University, cla18@humboldt.edu

Follow this and additional works at: http://digitalcommons.wayne.edu/jmasm

Part of the Applied Statistics Commons, Social and Behavioral Sciences Commons, and the Statistical Theory Commons

\section{Recommended Citation}

Aberson, Christopher (2015) "SPSS Programs for Addressing Two Forms of Power for Multiple Regression Coefficients," Journal of Modern Applied Statistical Methods: Vol. 14 : Iss. 1 , Article 21.

DOI: $10.22237 /$ jmasm/1430454000

Available at: http://digitalcommons.wayne.edu/jmasm/vol14/iss1/21 


\title{
JMASM Statistical Software Applications and Review SPSS Programs for Addressing Two Forms of Power for Multiple Regression Coefficients
}

\author{
Christopher Aberson \\ Humboldt State University \\ Arcata, California
}

This paper presents power analysis tools for multiple regression. The first takes input of correlations between variables and sample size and outputs power for multiple predictors. The second addresses power to detect significant effects for all of the predictors in the model. Both employ user-friendly SPSS Custom Dialogs.

Keywords: $\quad$ Power, sample size, simulation, SPSS, multiple regression, Power(all)

\section{Introduction}

Power analysis came to prominence with Jacob Cohen's seminal work on the topic (e.g., Cohen, 1988). Since that time, an extensive literature and several software packages and other resources focused on power (e.g., PASS, nQuery, Sample Power, G*Power, PiFace) emerged. Despite these advances, surveys across fields such as abnormal psychology (e.g., Sedlemeier \& Gigerenzer, 1989), consulting, clinical, and social psychology (Rossi, 1990), and neuroscience (Button et al., 2013) suggest that low power remains common in published literature.

One explanation for the persistence of underpowered studies, suggested by Cohen is that "researchers find too complicated ... reference material for power analysis (1992, p. 156)." The development of software approaches for power analysis allows researchers to move beyond some of the difficulties in understanding power analysis for many designs. With regard to power analyses for multiple regression designs, many approaches exist for estimating adequate

Christopher Aberson is Professor of Psychology. Email him at: cla18@humboldt.edu. 


\section{SPSS TOOLS FOR MULTIPLE REGRESSION POWER}

power for multiple $R^{2}$ (often termed $R^{2}$ model) based on considerations such as the number of predictors and sample size (see Algina \& Olejnik, 2003; Dunlap, Xin, \& Myers, 2004; Krishnamoorthy \& Xia, 2008; Mendoza \& Stafford, 2001; Murphy \& Myors, 2004; Shieh \& Kung, 2007).

Although many tools exist for power analyses focused on $R^{2}$ model, power analyses focused on multiple regression coefficients remains challenging. Existing resources for detecting power for coefficients are of limited utility, as most require input of complicated statistical values. For example, G*Power (Faul, Erdfelder, Buchner, \& Lang, 2009) provides protocols to address power for an individual predictor. This approach is accurate but requires that users input either partial $R^{2}$ or its components. The partial $R^{2}$ is a function of the proportion of variance uniquely explained by the predictor (squared semi-partial correlation) and the variance explained in the dependent measure by the other predictors in the model. This value is not particularly intuitive, nor is it commonly provided by most commercial packages. Similarly, the PiFace regression applet (Lenth, 20069) also provides a complex approach that requires entry of the variance inflation factor (VIF) and several other values. The VIF is an index of overlap between predictors. Although common to most statistical packages, the VIF statistic, reflecting one divided by the residual variance from an analysis regressing the predictor of interest on the other predictors, is also not intuitive to most researchers. Additionally, both approaches require separate estimates for each predictor of interest. That is, to get accurate power estimates, users must repeat a complex set of calculations for each predictor. It is my impression that most researchers find it difficult to estimate values such as partial $R^{2}$ and VIF accurately for power analysis. These tools are well designed and accurate; however, the complexity of the required inputs limits their usability.

The estimates required by these protocols are "endpoint" values. Endpoint values are statistical values that require extensive computation for accurate estimation. Endpoint values such as the partial $R^{2}$ and VIF are a function of the correlation between the predictors and the dependent variable and the strength of correlations between the predictor of interest and other predictors in the model (i.e., a correlation matrix). Although partial $R^{2}$ and VIF are difficult to estimate, the zero-order correlations that produce these values are not. A researcher basing power analyses on previous work on the variables of interest is far more likely to find presentation of zero-order correlations between variables than VIF or partial $R^{2}$ statistics. For this reason, the protocols introduced in this paper focus on input of correlations as the primary statistical values for power analysis. 


\section{CHRISTOPHER ABERSON}

Another explanation for low power in designs with multiple predictors is a lack of attention to power for detecting a set of outcomes. Researchers using multiple regression models with three predictors commonly want to detect significant coefficients for all of the predictors. However, applications of power analyses for designs with multiple predictors typically yield an estimate of power for each predictor (e.g., Aberson, 2010), but not power to detect all of them in the same study. Problematically, power to detect multiple effects differs considerably from power for individual effects. In most research situations, power to detect multiple effects is considerably lower than the power for individual effects. The lack of attention to this form of power is a likely source underpowered research in the behavioral sciences (Maxwell, 2004).

The paper introduces tools to calculate simultaneous power estimates for two or more multiple regression coefficients (MRPower), power for detecting significant effects on all coefficients in a model (MRPower Simulate), and presents analyses using a series of SPSS Custom Dialogs based on the syntax found in Appendices $\mathrm{A}$ and $\mathrm{B}$ and available from http://users.humboldt.edu/chris.aberson/Index.html. All tools require entry of zero-order correlations with several additional optional values.

\section{Equations for power calculations}

Power for multiple regression coefficients is a function of the regression coefficient and its standard error with these values being a function of the correlations among variables in the model. The calculation of the standardized regression coefficient (Eq.1) involves both the correlations between the predictors (represented with numbers) and the criterion or dependent variable (represented with $y$ ). In this equation, $r_{y 1}$ is the correlation between the first predictor and the $d v, r_{y 2}$ is the correlation between the second predictor and the $d v$, and $r_{12}$ is the correlation between the first predictor and the second predictor.

$$
b_{y 1.2}^{*}=\frac{r_{y 1}-r_{y 2} r_{12}}{1-r_{12}^{2}}
$$

A simplified explanation of Equation 1 is that the coefficient is larger when correlations between the predictor and $D V$ are large but becomes smaller when predictors correlate in the same direction as in the second predictor- $d v$ relationship. In terms of the influence on power analysis, larger coefficients produce more power. 


\section{SPSS TOOLS FOR MULTIPLE REGRESSION POWER}

The standard error of the standardized regression coefficient (Eq. 2) is a function of the total variance explained by the two predictors in the analyses (often termed $R^{2}$ model, represented as $R_{y .12}^{2}$ ) and the squared correlation of the two predictors $\left(r_{12}^{2}\right)$. The standard error is smaller when the variables explain more variance, when the correlation between predictors is smaller, and when sample size $(n)$ is larger.

$$
s e_{b^{*}}=\sqrt{\frac{1-R_{y .12}^{2}}{\left(1-r_{12}^{2}\right) *(n-3)}}
$$

Calculation of the standard error requires $R^{2}$ for a model with all the predictors (Eq. 3). This value increases as correlations between predictors and the $D V$ increase and gets smaller as correlations between predictors rise, provided that correlations all run in the same direction.

$$
R_{y .12}^{2}=\frac{r_{y 1}^{2}+r_{y 2}^{2}-2 r_{y 1} r_{y 2} r_{2}}{1-r_{12}^{2}}
$$

The ratio of coefficient to standard error produces the non-centrality parameter $(\delta)$. Larger $\delta$ values represent more power. This value allows for calculation of power. Power calculations require application of non-central distribution probability density functions that are beyond the scope of simple calculations. However, SPSS and other packages provide the calculation (see next section for application).

$$
\delta=\frac{b_{y 1.2}^{*}}{s e_{b^{*}}}
$$

These formulae demonstrate several important concepts relevant to power analysis with multiple predictors. First, larger regression coefficients (i.e., larger effect size) promote more power. Larger coefficients result from stronger correlations between predictors and the $D V$. Correlation between predictors drives coefficient size downward and thus reduces power. Broadly this means that collinearity (or with three or more predictors, multicollinearity) reduces statistical power. 


\section{CHRISTOPHER ABERSON}

\section{Power for two predictors}

This section presents calculations of power for a two predictor example and then introduces the MRPower SPSS program to perform power calculations.

\section{Calculation example}

This example predicts voting intentions relevant to a hypothetical proposition to continue or discontinue affirmative action (on a scale where $0=$ Absolutely will vote to eliminate to $10=$ Absolutely will vote to continue) from beliefs that AA is fair and rejection of the merit principal. For the predictors, higher scores mean more fairness and stronger perceptions that merit should not be the only consideration in hiring. Based on earlier work, the example uses for $r_{y 1}=.5$ (the correlation between fairness and intention), $r_{y 2}=.4$ (the correlation between merit and intention), and $r_{12}=.3$ (the correlation between fairness and merit). The section that follows demonstrates calculation of power for a sample of $n=50$.

$$
\begin{aligned}
R_{y .12}^{2} & =\frac{r_{y 1}^{2}+r_{y 2}^{2}-2 r_{y 1} r_{y 2} r_{12}}{1-r_{12}^{2}}=\frac{.5^{2}+.4^{2}-2(.5)(.4)(.3)}{1-.3^{2}}=.3187 \\
b_{y 1.2}^{*} & =\frac{r_{y 1}-r_{y 2} r_{12}}{1-r_{12}^{2}}=\frac{.5-\left(.4^{*} .3\right)}{1-.3^{2}}=.4176 \\
b_{y 2.1}^{*} & =\frac{r_{y 2}-r_{y 1} r_{12}}{1-r_{12}^{2}}=\frac{.4-\left(.5^{*} .3\right)}{1-.3^{2}}=.2747 \\
s e_{b^{*}} & =\sqrt{\frac{1-R_{y .12}^{2}}{\left(1-r_{12}^{2}\right) *(n-3)}}=\sqrt{\frac{1-.3187}{\left(1-.3^{2}\right)(50-3)}}=.1262 \\
\delta_{1} & =\frac{b_{y 1.2}^{*}}{s e_{b^{*}}}=\frac{.4176}{.1262}=3.309 \\
\delta_{2} & =\frac{b_{y 2.1}^{*}}{s e_{b^{*}}}=\frac{.2747}{.1262}=2.177
\end{aligned}
$$

With alpha $=.05$, Power $x_{1}=.90$ (fairness) and Power $x_{2}=.57$ (merit). To obtain these values, provide SPSS with the following syntax for the first predictor: Compute Power $=1$ - NCDF.T (2.012,47,3.309). The value 2.012 represents the critical value of $t$ for rejection of the null, using two-tailed $\alpha=.05$. The value 47 represents degrees of freedom and 3.309 is $\delta$. 


\section{SPSS TOOLS FOR MULTIPLE REGRESSION POWER}

\section{Two predictor power using MRPower}

The MRPower Two dialog provides a user-friendly interface that takes input of correlation values and sample size and returns power for each coefficient and $R^{2}$ model. The interface also allows users to enter labels for each variable, desired Type I error level for tests of the model and for coefficients, and the directory for files generated by the analyses. These values are optional. Figure 1 demonstrates entry of values into MRPower Two. Figure 2 presents the output from the dialog, yielding values consistent with calculations as well as an estimate for $R^{2}$ model power. The output provides power for all coefficients simultaneously. To obtain a desired level of power, increase sample size until reaching the target value. Power $\geq .80$ for both coefficients requires a sample of 83 , whereas Power $\geq .90$ for both coefficients requires 110 participants.

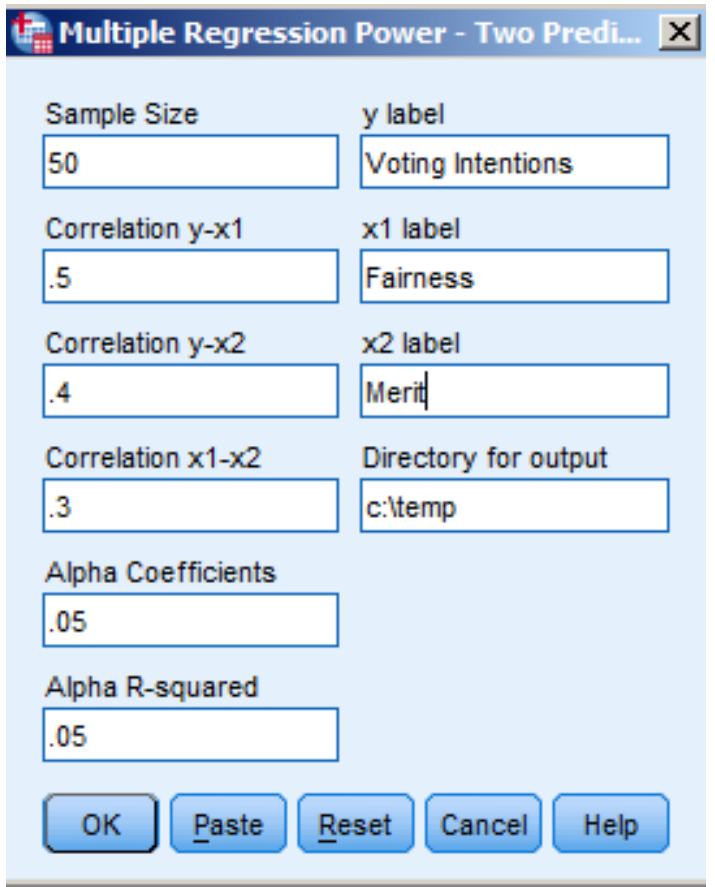

Figure 1. MRPower two interface demonstrating calculation of power for two individual predictors. 


\section{CHRISTOPHER ABERSON}

\begin{tabular}{|r|r|r|r|}
\hline Sample Size & $\begin{array}{c}\text { Power R- } \\
\text { squared }\end{array}$ & $\begin{array}{c}\text { Power } \\
\text { Fairness }\end{array}$ & Power Merit \\
\hline 50 & .8275 & .8998 & .5683 \\
\hline
\end{tabular}

Figure 2. MRPower two output for the analysis specified in Figure 1

\section{Models with three predictors}

Calculations for two predictor models are relatively straightforward. Models with three or more predictors require approaches that are substantially more complex. For three or more predictions, calculations involve matrix inversion and other approaches that likely go beyond the backgrounds of most researchers (see Cohen, Cohen, West, \& Aiken, 2003 for calculator approaches). The syntax and custom dialogs presented in this paper provide researchers with tools to obtain power estimates for multiple regression designs with three variables through a simple extension of the approach employed in the two predictor section. Although not demonstrated in this paper, dialogs for four through ten predictors (named MRPower Four, MRPower Five, etc.) are in development.

\section{Three predictors with MRPower}

The example that follows demonstrates use of MRPower to determine adequate sample size. This example takes results from Aberson (2007) and uses those values to determine power for a new study involving three predictors of general attitudes toward affirmative action. The predictors are diversity valuation, belief in the need for affirmative action, and personal experiences of discrimination with their expected population correlations shown in Table 1.

Figure 3 demonstrates the MRPower Three interface. In this example, to obtain power of .80 or greater for each predictor requires a sample size of 129 . Specifically, as shown in Figure 4, the analysis reports power of .94 for diversity, .82 for belief in need, and .80 for experience of discrimination.

Table 1. Correlations between variables in three predictor example.

\begin{tabular}{rrrr} 
& General Policy & Diversity & Belief in Need \\
\hline General & $.45\left(r_{y 1}\right)$ & & \\
Diversity & $-.39\left(r_{y 2}\right)$ & $-.42\left(r_{12}\right)$ & \\
Belief in Need & $-.31\left(r_{y 3}\right)$ & $-.22\left(r_{13}\right)$ & $.11\left(r_{23}\right)$ \\
Exp of Disc &
\end{tabular}




\section{SPSS TOOLS FOR MULTIPLE REGRESSION POWER}

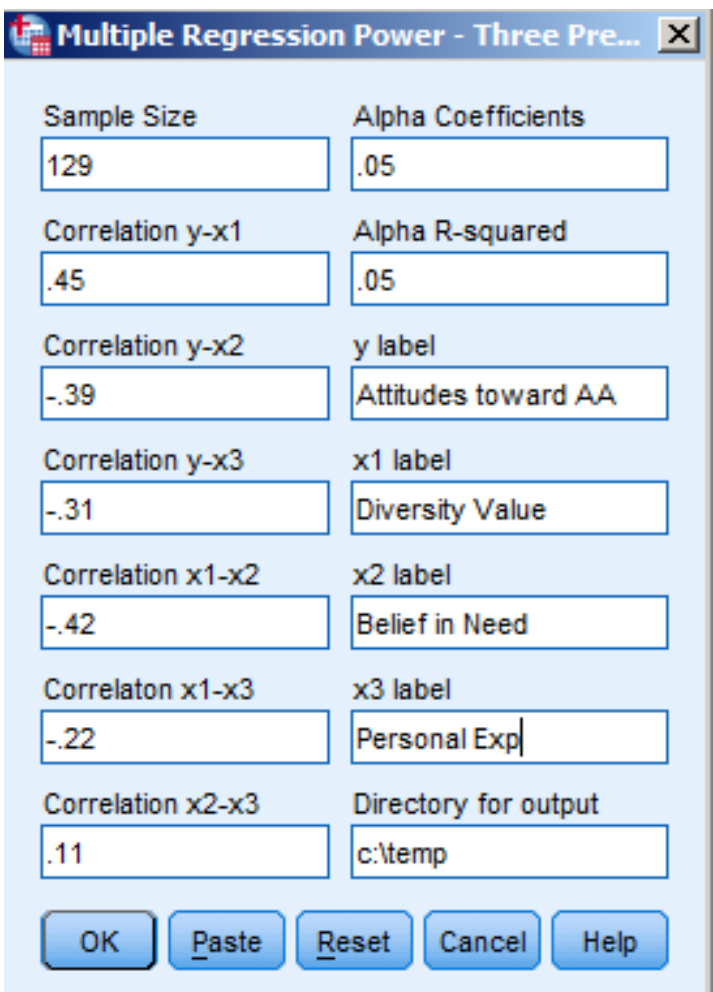

Figure 3. MRPower Three interface demonstrating calculation of individual power for three predictors.

\begin{tabular}{|r|r|r|r|r|}
\hline Sample Size & $\begin{array}{c}\text { Power R- } \\
\text { squared }\end{array}$ & $\begin{array}{c}\text { Power } \\
\text { Diversity } \\
\text { Value }\end{array}$ & $\begin{array}{c}\text { Power Belief } \\
\text { in Need }\end{array}$ & $\begin{array}{c}\text { Power } \\
\text { Personal Exp }\end{array}$ \\
\hline 129 & .9481 & .9444 & .8194 & .8005 \\
\hline
\end{tabular}

Figure 4. MRPower Three output for the analysis specified in Figure 3.

\section{Power for detecting significant effects for all predictors in the model}

Often researchers using multiple regression want to detect significant effects for all of the predictors in a model. However, existing power analysis approaches only address power for individual predictors. This section details how power to detect effects for all of the predictors in a model differs from power to detect individual effects and present tools for addressing this form of power. The 


\section{CHRISTOPHER ABERSON}

primary issue relevant to detecting significant effects for multiple predictor variables is the role of Beta error inflation (or Familywise Beta error; see Maxwell, 2004 for a technical discussion). This issue is similar to inflation of $\alpha$ or Type I error. When conducting multiple significance tests, Type I error rates for the family of tests (a.k.a., familywise alpha) increase. Equation 5 provides an estimate of familywise $\alpha$ error for multiple comparisons and is the conceptual basis for development of tests such as the Bonferroni adjustment. According to the formula, with three tests using a pairwise alpha $\left(\alpha_{p w}\right)$ of .05, familywise alpha $\left(\alpha_{f w}\right)$ is .14.

$$
\alpha_{f w}=1-\left(1-\alpha_{p w}\right)^{c}
$$

The same process is at work with regard to the familywise probability of making a $\beta$ or Type II error (Equation 6), a value referred as $\beta_{f w}$ throughout the paper. For example, take a study designed for $\beta$ of .20 (called $\beta_{\text {ind }}$ for Beta individual) for each of its three predictors (a.k.a., Power $=.80$ for each predictor). The likelihood of making a single $\beta$ error among those three tests is substantially higher than the error rate of .20 for the individual tests. Just as with $\alpha$ error, multiple tests inflate the chances to make a single $\beta$ error among a set of significance tests. The $\beta_{f w}$ value easily converts to power to detect all of the effects in the design by taking $1-\beta_{f w}$. Throughout the paper, this value is referred to as Power(All).

$$
\beta_{f w}=1-\left(1-\beta_{\text {ind }}\right)^{c}
$$

Table 2. Familywise Type II error (Beta) rates for predictors using $\beta_{p w}=.20($ Power $=.80)$

\begin{tabular}{rrr} 
Number of Predictors & $\beta_{f w}$ & Power(All) \\
\hline 2 & .360 & .640 \\
3 & .488 & .512 \\
4 & .590 & .410 \\
5 & .672 & .328 \\
6 & .738 & .262 \\
7 & .790 & .210 \\
8 & .832 & .168 \\
9 & .866 & .134 \\
10 & .893 & .107 \\
\hline
\end{tabular}

* Note. All predictors uncorrelated. This table is not accurate for correlated predictors. 


\section{SPSS TOOLS FOR MULTIPLE REGRESSION POWER}

Table 2 shows $\beta_{f w}$ and Power(All) for two through 10 predictors. One clear result here is that in models with four predictors or more, if the researcher designs for Power $=.80$ for each individual predictor, the study will more likely than not fail to find significance on at least one of the predictors. This table is useful for a conceptual understanding of $\beta_{f w}$, however these results (and Eq. 6) are only accurate for calculations where all tests have the same power and predictors are uncorrelated.

\section{Power(All) for designs with correlated predictors}

Calculation of $\beta_{p w}$ and Power(All) is straightforward for situations where predictors are uncorrelated. However, in most multiple regression applications predictors do correlate. How this influences Power(All) is a function of the strength and direction of correlations between predictors. Broadly, when predictors correlate positively with each other, Power(All) decreases. If predictors negatively correlate, Power(All) increases.

Calculations of Power(All) given correlated predictors are best handled by simulation. Simulations draw a large number of independent samples (e.g., 10,000 ) from a population with parameters used in the power analysis (defined by a correlation matrix). From those samples, count how many allow rejection of null hypotheses relevant to all of the predictors in the study. The proportion of samples producing results allowing for rejection of all hypotheses reflects Power(All).

Table 3 demonstrates the impact of predictor correlations on Power(All) for a two predictor model. Power for each predictor is constant across each situation at .80 (the correlation between the predictors and $D V$ changes to create this level of power) and the sample size is 50. The Reject All column reflects Power(All) estimates derived by simulation of 10,000 samples drawn from a population with the given correlations. Since this approach is empirical, there is some deviation from theoretical probabilities. For example, Power(All) for two predictors with Power $=.80$ and no correlation between predictors is theoretically .64 . The simulation provides a value of .6348 . Although not exact with 10,000 replications, the simulated values provide a clear demonstration of the patterns of expected results. The range of values for Power(All) is roughly .59 to .72 with more power generated as correlations between predictors move from strongly positive to strongly negative.

These values suggest that negative correlations between predictors are advantageous. However, is important to recognize that it is unlikely to find 


\section{CHRISTOPHER ABERSON}

predictors that correlate strongly in the negative direction when both predictors have a consistent (i.e., all positive or all negative) relationships with the $D V$.

Table 3. Power(All) for two predictors with power $=.80$ and varying levels of correlation.

\begin{tabular}{rrrrr}
$\begin{array}{r}\text { Correlation between } \\
\text { predictors }\end{array}$ & $\begin{array}{r}\text { Required } x-y \\
\text { correlations }\end{array}$ & $\begin{array}{r}\text { Reject } \\
\text { None }\end{array}$ & $\begin{array}{r}\text { Reject } \\
\text { One }\end{array}$ & $\begin{array}{r}\text { Reject } \\
\text { All }\end{array}$ \\
\hline-.80 & .1274 & .1294 & .1492 & .7214 \\
-.60 & .1891 & .1074 & .2029 & .6897 \\
-.40 & .2445 & .0816 & .2458 & .6726 \\
-.20 & .2999 & .0564 & .2912 & .6524 \\
.00 & .3594 & .0463 & .3189 & .6348 \\
.20 & .4266 & .0279 & .3518 & .6203 \\
.40 & .5070 & .0190 & .3708 & .6102 \\
.60 & .6102 & .0102 & .3864 & .6034 \\
.80 & .7561 & .0033 & .4107 & .5860 \\
\hline
\end{tabular}

* Note. Required $x-y$ correlation is the correlation between each predictor and the $d v$ to produce Power $=.80$ with $\mathrm{n}=50$.

Table 4 demonstrates Power(All) for models with three predictors. In each situation, Power $=.80$ for each predictor and the sample size is 100 . One striking finding here is that Power(All) can be as low as .44 for a model with strongly correlated predictors, despite the relatively high level of power for individual predictors. As with the two predictor model, Power(All) rises as correlations among predictors move from positive to negative. However, Power(All) tends to be smaller with more predictors. For two predictors, Power(All) ranges from .59 to .72 whereas with three predictors, Power(All) goes from .44 to .64 .

Table 4. Power(All) for three predictors with power $=.80$ and varying levels of correlation.

\begin{tabular}{|c|c|c|c|c|c|}
\hline $\begin{array}{r}\text { Correlation } \\
\text { between } \\
\text { predictors }\end{array}$ & $\begin{array}{r}\text { Required } x-y \\
\text { correlations }\end{array}$ & Reject None & Reject One & Reject Two & Reject All \\
\hline-.80 & $\mathrm{n} / \mathrm{a}$ & & & & \\
\hline-.60 & $\mathrm{n} / \mathrm{a}$ & & & & \\
\hline-.40 & .0804 & .0793 & .1030 & .1800 & .6377 \\
\hline-.20 & .1692 & .0268 & .1129 & .3046 & .5557 \\
\hline .00 & .2583 & .0091 & .1005 & .3678 & .5226 \\
\hline .20 & .3569 & .0033 & .0892 & .4251 & .4824 \\
\hline .4 & .4703 & .0008 & .0678 & .4681 & .4633 \\
\hline 6 & .6057 & .0001 & .0506 & .5000 & .4493 \\
\hline .8 & .7747 & .0000 & .0435 & .5211 & .4354 \\
\hline
\end{tabular}

* Note. Required $x-y$ correlation is the correlation between each predictor and the $d v$ to produce Power $=.80$ with $n=100$. 


\section{SPSS TOOLS FOR MULTIPLE REGRESSION POWER}

Also of note is that some values in Table 4, represented as n/a, are not possible. For example, there is no predictor- $D V$ correlation where it is possible to have correlations of -.60 or -.80 between the predictors (given $n=100$ ). Additionally, models with substantial positive correlations among multiple predictors likely violate regression assumptions regarding multicollinearity.

\section{MRPower Simulate dialogs}

The previous section demonstrated how correlations between predictors impact Power(All). However, the values presented in those tables are limited as they reflect situations wherein correlations between predictors and power for individual predictors are constant. Practically predictors might show different levels of power and varying levels of correlation. The MRPower Simulate dialogs allow for such input and address Power(All) for designs with two to ten predictors.

In the example from the previous section, power exceeded .80 for three predictors with a sample of 129 . However, power for detecting significant effects for all three predictors in the same sample [termed Power(All)] is likely substantially smaller. The MRPower Simulate dialog creates a population based on user-supplied correlations. Next, the program takes a sample of size $n$ from the population ( $n$ is specified by the user) and generates an analysis predicting the $D V$ from the set of IVs for that sample. The results of the analysis are output to a datafile (stored in the directory c: Itemp as a default). The program repeats this process 10,000 times. Finally, the program compiles rejection rates and provides output representing power for individual coefficients (total times rejecting null divided by total number of replications) and power for rejecting zero to all coefficients.

The number of replications and population size are modifiable. Although population is theoretically infinite, a finite population of 100,000 is, for most purposes, large enough to produce an accurate result. In testing the dialog, there was little difference between the default settings and simulations using larger populations (e.g., 10 million) and more replications (e.g., 100,000). However, more replications substantially increased processing time. If sample sizes begin to approach even a small percentage of population size, it would likely be beneficial to increase the population size. For quick analyses (e.g., trying to determine whether the sample size for Power(All) $=.80$ is closer to 300 than 400), replications might be reduced initially then increased in subsequent runs for a precise result. 


\section{CHRISTOPHER ABERSON}

\section{MRPower Simulate example.}

Figure 5 demonstrates the MRPower Simulate dialog using a sample of 129 and the correlations from Table 1. As shown in Figure 6, this analysis generates Power(All) $=.6056$ to detect all three effects in the same model. The output also indicates the number of samples rejecting null hypotheses for zero, one, or two coefficients. On a positive note, the likelihood of finding no significant effects is .0001 .

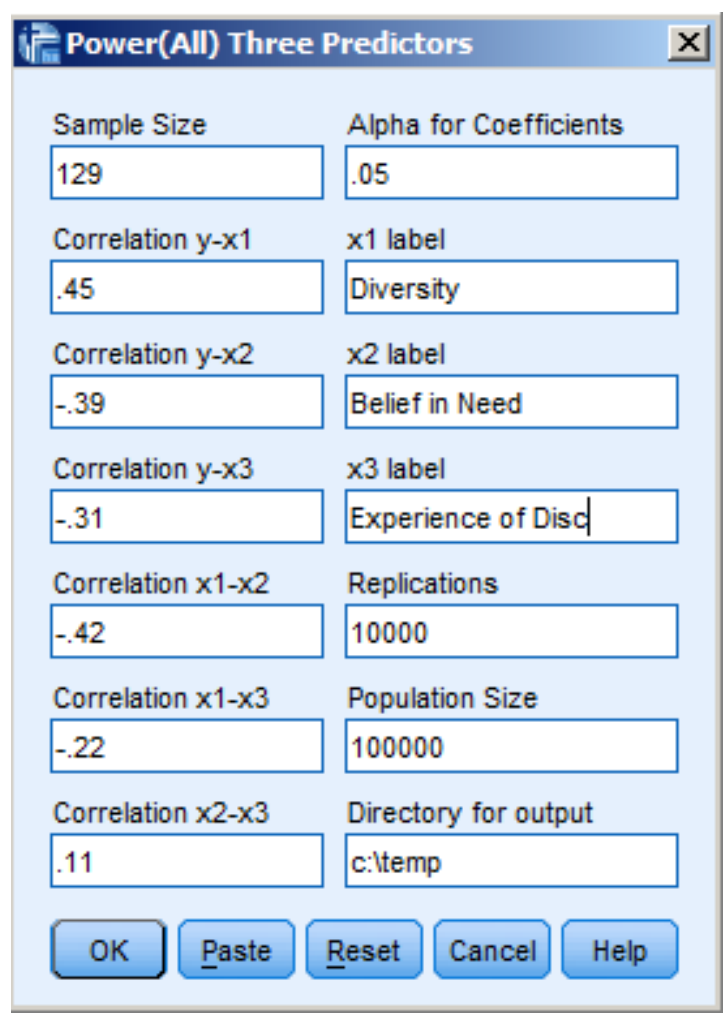

Figure 5. MRPower Simulate three interface for calculation of Power(All).

Figure 6 also presents power for each individual predictor. This value is the number of times rejecting the null for the predictor over total number of replications. These values provide a useful check against the results of the MRPower Three dialog. In this case, power for Diversity (.9387 vs. .9444), Power for Belief in Need (.8154 vs. .8194), and Power for Personal Experience (.8039 vs. .8005) are all consistent with the MRPower estimates. If these values 


\section{SPSS TOOLS FOR MULTIPLE REGRESSION POWER}

are not consistent, it suggests incorrect specification of the parameters of the model (i.e., something not entered correctly in the dialog).

\begin{tabular}{|c|c|c|c|c|c|c|}
\hline & & & & \multicolumn{3}{|c|}{ Power for Individual Coefficients } \\
\hline \multicolumn{4}{|c|}{ Number of Coefficients Rejected } & & & Power \\
\hline .00 & 1.00 & 2.00 & 3.00 & Diversity & $\begin{array}{l}\text { in Need } \\
\text { int }\end{array}$ & $\begin{array}{l}\text { Experlence of } \\
\text { Disc }\end{array}$ \\
\hline $0.01 \%$ & $4.74 \%$ & $34.69 \%$ & $60.56 \%$ & 93.87 & 81.54 & 80.39 \\
\hline
\end{tabular}

Figure 6. MRPower Simulate three output for Power(All) and individual predictors given specification from Figure 5.

A final question is how large a sample is necessary for Power(All) of a specific value (e.g., .80). Using the simulation tool, Power(All) hits .80 with $n=171$. For $n=171$, power for the individual predictors are $.98, .91$, and .90 respectively. This represents an increase of roughly one-third of the original sample size estimate.

\section{References}

Aberson, C. L. (2007). Diversity, merit, fairness, and discrimination beliefs as predictors of support for affirmative action policy actions. Journal of Applied Social Psychology, 37(10), 2451-2474. doi:10.1111/j.1559-1816.2007.00266.x

Aberson, C. L. (2010). Applied power analysis for the behavioral sciences. New York: Psychology Press.

Algina, J., \& Olejnik, S. (2003). Sample size tables for correlation analysis with applications in partial correlation and multiple regression analysis.

Multivariate Behavioral Research, 38(3), 309-323.

doi:10.1207/S15327906MBR3803_02

Button, K. S., Ioannidis, J. P. A., Mokrysz, C., Nosek, B. A., Flint, J., Robinson, E. S. J., \& Munafò, M. R. (2013). Power failure: Why small sample size undermines the reliability of neuroscience. Nature Reviews Neuroscience, 14, 365-376. doi:10.1038/nrn3475

Cohen, J. (1988). Statistical power analysis for the behavioral sciences $\left(2^{\text {nd }}\right.$ ed.). Hillsdale, NJ: Lawrence Erlbaum Associates.

Cohen, J. (1992). A power primer. Psychological Bulletin, 112(1), 155-159. doi:10.1037/0033-2909.112.1.155 


\section{CHRISTOPHER ABERSON}

Cohen, J., Cohen, P., West, S. G., \& Aiken, L. S. (2003). Applied multiple regression/Correlation analysis for the behavioral sciences ( $3^{\text {rd }}$ ed.). Hillsdale, $\mathrm{NJ}$ : Lawrence Erlbaum Associates.

Dunlap, W. P., Xin, X., \& Myers, L. (2004). Computing aspects of power for multiple regression. Behavior Research Methods, Instruments \& Computers, 36(4), 695-701. doi:10.3758/BF03206551

Faul, F., Erdfelder, E., Buchner, A., \& Lang, A. G. (2009). Statistical power analyses using $\mathrm{G}^{*}$ Power 3.1: Tests for correlation and regression analyses. Behavior Research Methods, 41(4), 1149-1160. doi:10.3758/BRM.41.4.1149

Krishnamoorthy, K, \& Xia, Y. (2008). Sample size calculation for estimating or testing a nonzero squared multiple correlation coefficient. Multivariate Behavioral Research, 43(3), 382-410. doi:10.1080/00273170802285727

Lenth, R. V. (2006-9). Java applets for power and sample size [Computer software]. Retrieved from http://www.stat.uiowa.edu/ rlenth/Power.

Maxwell, S. E. (2004). The persistence of underpowered studies in psychological research: Causes, consequences, and remedies. Psychological Methods, 9(2), 147-163. doi:10.1037/1082-989X.9.2.147

Mendoza, J. L., \& Stafford, K. L. (2001). Confidence interval, power calculation, and sample size estimation for the squared multiple correlation coefficient under the fixed and random regression models: A computer program and useful standard tables. Educational and Psychological Measurement, 61(4), 650-667. doi:10.1177/00131640121971419

Murphy, K. R., \& Myors, B. (2004). Statistical power analysis: A simple and general model for traditional and modern hypothesis tests (2nd ed.). Hillsdale, NJ: Laurence Erlbaum Associates.

Rossi, J. S. (1990). Statistical power of psychological research: What have we gained in 20 years? Journal of Consulting and Clinical Psychology, 58(5), 646-656. doi:10.1037/0022-006X.58.5.646

Sedlmeier, P., \& Gigerenzer, G. (1989). Do studies of statistical power have an effect on the power of studies? Psychological Bulletin, 105(2), 309-316. doi:10.1037/0033-2909.105.2.309

Shieh, G., \& Kung, C. F. (2007). Methodological and computational considerations for multiple correlation analysis. Behavior Research Methods, 39(4), 731-734. doi:10.3758/BF03192963 


\section{SPSS TOOLS FOR MULTIPLE REGRESSION POWER}

\section{Appendix A}

\section{MRPower Three Syntax}

*Values noted with \%\% are user supplied values from the dialog. For example if $\mathrm{n}$

$=60$ is entered $*_{\text {in }}$ the dialog, the $\%$ n\% is replaced by 60 for analyses.

*OMS command suppresses output

OMS SELECT ALL

/DESTINATION VIEWER=NO.

*Creates correlation matrix for analysis

*Means are set at $1,2,3$, and 4 to facilitate SPSS processing.

*Os sometimes cause SPSS to terminate

MATRIX DATA VARIABLES $=$ ROWTYPE_ $y \times 1 \times 2 \times 3$.

BEGIN DATA

Mean 12334

STDEV $\begin{array}{lllll}1 & 1 & 1 & 1\end{array}$

N \%\%n\% \%\%n\% \%\%n\% \%n\%\%

Corr 1

Corr \% \% ry $1 \% \% 1$

Corr \% \% ry $2 \% \%$ \% $12 \% \% \quad 1$

Corr \% \%ry3\%\% \% $\%$ r $13 \% \% \quad \%$ r23\% 1

END DATA.

DATASET CLOSE \%\%dir\% \resultsC.sav.

*Captures coefficient and $\mathrm{R}^{2}$ values for power calculations OMS SELECT TABLES

/destination format $=$ sav numbered $=$ "Table_Number" outfile = "\%dir\% \resultsC.sav"

/if commands $=$ ['regression'] subtypes $=$ ['Coefficients']

/tag = "reg".

OMS SELECT TABLES

/destination format $=$ sav numbered $=$ "Table_Number" outfile = "\%dir\%\resultsC.sav"

/if commands $=$ ['regression'] subtypes $=$ ['ANOVA']

/tag = "regF". 


\section{CHRISTOPHER ABERSON}

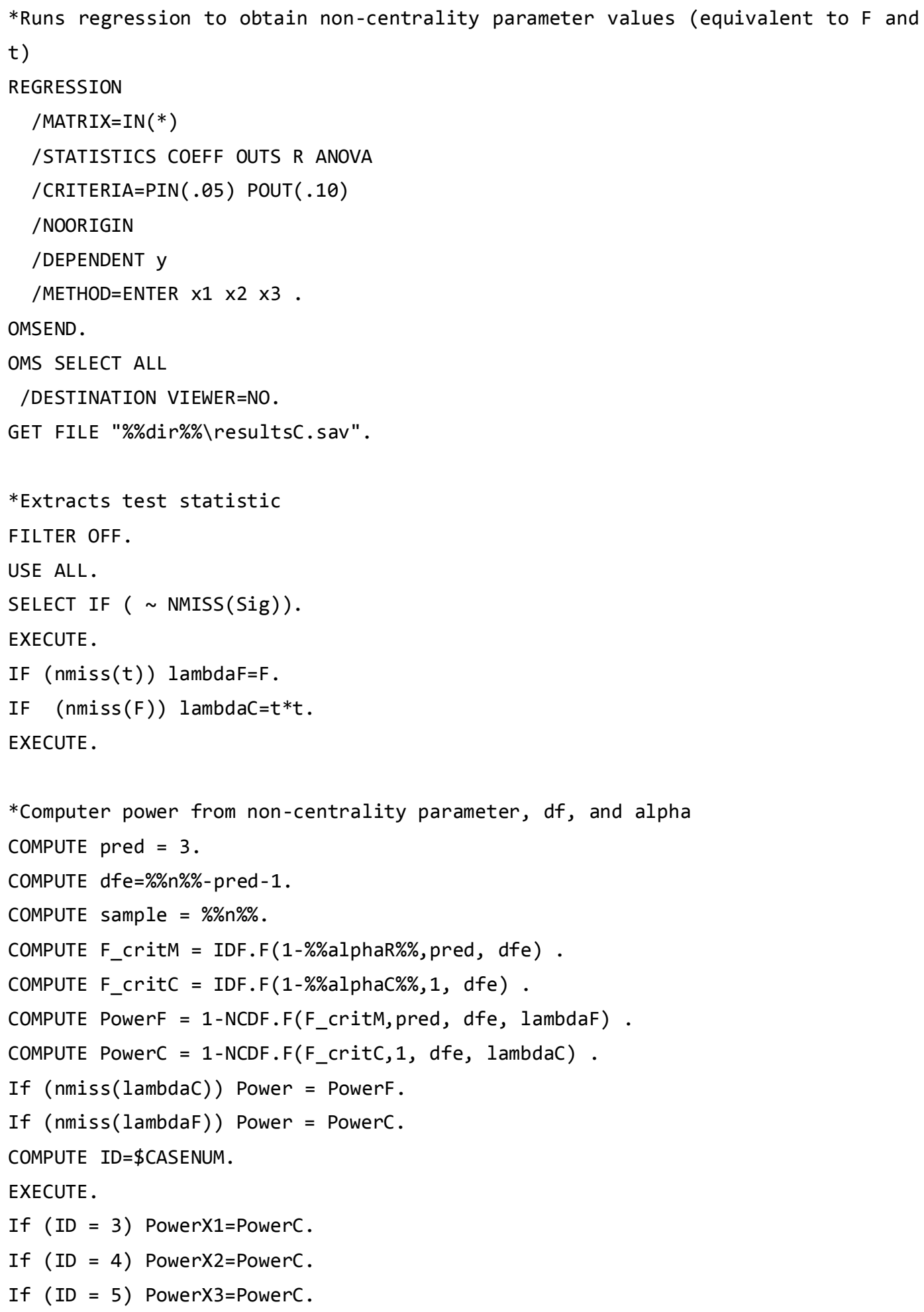




\section{SPSS TOOLS FOR MULTIPLE REGRESSION POWER}

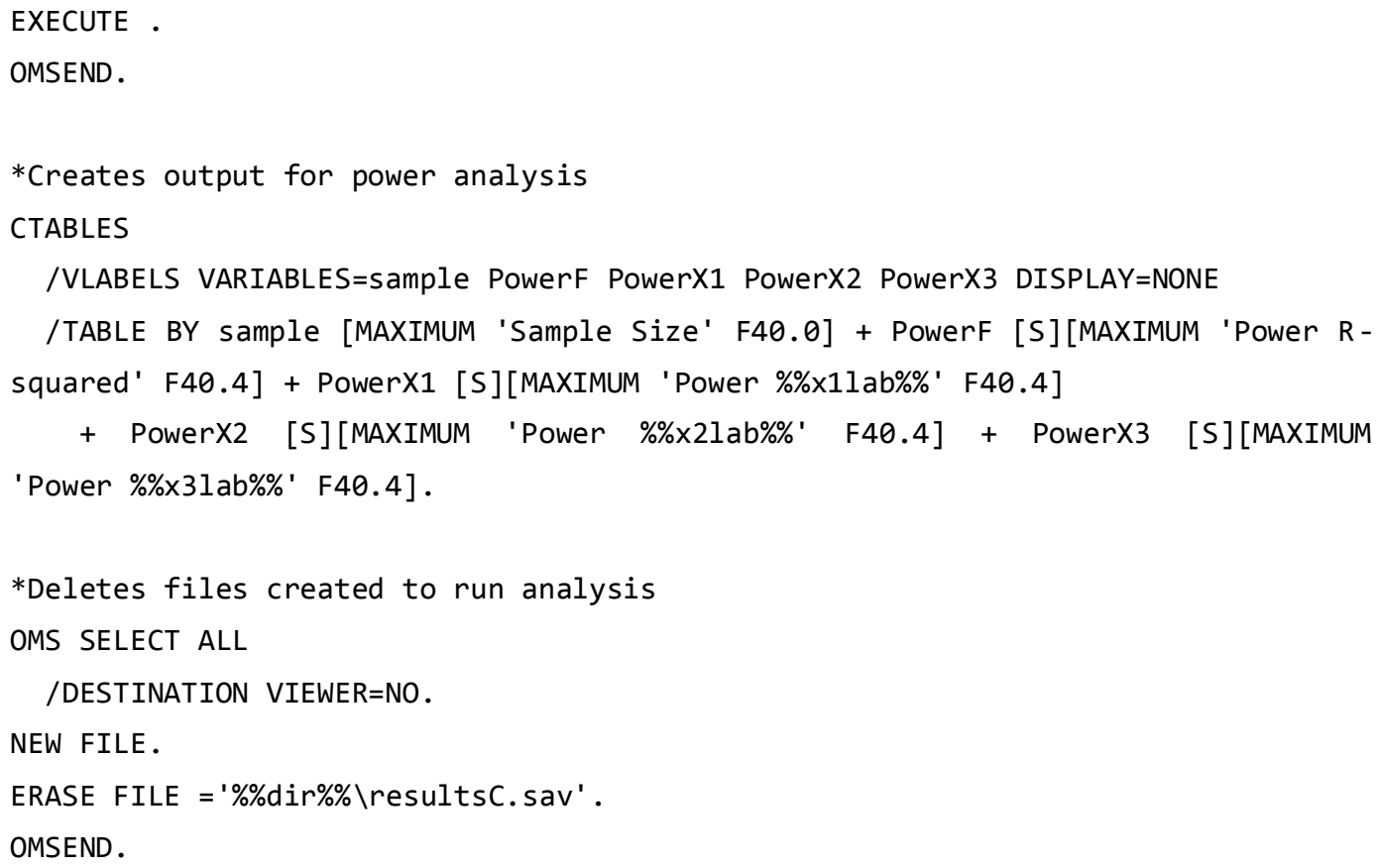




\section{CHRISTOPHER ABERSON}

\section{Appendix B}

\section{MRPower Simulate Three Syntax}

*Values noted with \%\% are user supplied values from the dialog.

*This command suppresses output

OMS SELECT ALL

/DESTINATION VIEWER=NO.

*The data generation approach used here modifies syntax presented in an IBM SPSS support

*file at http://WWW-01.ibm.com/support/docview.wss?uid=swg21480900 . Based on personal *correspondence and references to edstat-1 archives, I believe this approach was developed *by David Nichols.

matrix data variables $=\mathrm{v} 1$ to $\mathrm{v} 4$

/contents=corr .

begin data.

1

$\%$ ry $1 \% \% 1$

$\%$ ry $2 \% \% \quad \%$ r $12 \% \% \quad 1$

$\%$ \%ry3\%\% $\%$ r $13 \% \% \quad \%$ r23\%\% 1

end data.

save outfile='\%dir\%\\corrmat.sav'

/keep=v1 to $\mathrm{v} 4$.

*Generate raw data. Loop \# generates desired population size.

*Vector $\mathrm{x}()$ and $\#$ j reflect number of variables (1 $d v, 3$ predictors in this example)

new file.

input program.

loop \#i=1 to \%popsize\%\%.

vector $x(4)$.

loop $\# j=1$ to 4 .

compute $x(\# j)=r v \cdot \operatorname{normal}(0,1)$.

end loop.

end case.

end loop.

end file.

end input program. 


\section{SPSS TOOLS FOR MULTIPLE REGRESSION POWER}

execute.

*FACTOR procedure generates principal components, which will be uncorrelated and have *mean $\theta$ and standard deviation 1 for each variable.

factor $\operatorname{var}=x 1$ to $x 4$

/criteria=factors (4)

/save=reg (all z).

matrix.

get $z / v a r=z 1$ to $z 4$.

get $r /$ file $=\%$ dir\% $\$ corrmat.sav' .

compute out $=z^{*} \operatorname{chol}(r)$.

save out /outfile='\%dir\%\giant_datafile.sav' .

end matrix.

*End data generation portion

*Gets the generated data and test correlations.

get file='\%\%dir\%\giant_datafile.sav' .

*Rename variables

RENAME variables col1 = $\mathrm{y}$.

RENAME variables ( $c 012$ to col4=x1 to $x 3$ ).

COMPUTE ID $=\$$ CASENUM .

SAVE OUTFILE=' \%\%dir\% $\backslash$ giant_datafile.sav'

/COMPRESSED.

*This piece draws random samples of size $\mathrm{n}$. Creates number of samples equal to reps.

*Puts everything in one file then splits it by sample number INPUT PROGRAM .

LOOP SAMP=1 to \%reps\%.

LOOP $V=1$ to $\% \%$ \% .

COMPUTE ID=TRUNC(UNIFORM(\%popsize\%\%) $)+1$.

END CASE.

LEAVE SAMP.

END LOOP.

END LOOP.

END FILE. 


\section{CHRISTOPHER ABERSON}

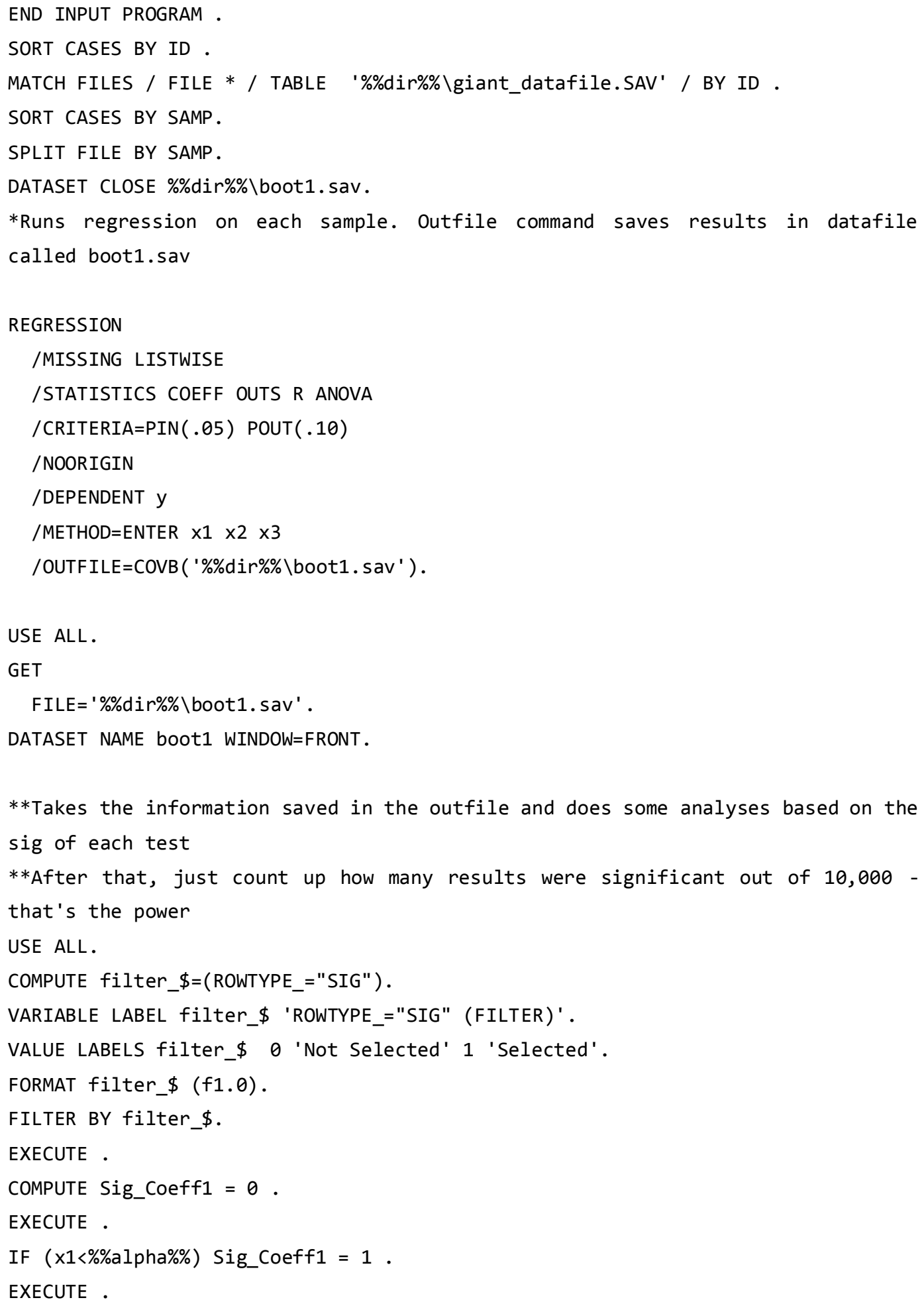




\section{SPSS TOOLS FOR MULTIPLE REGRESSION POWER}

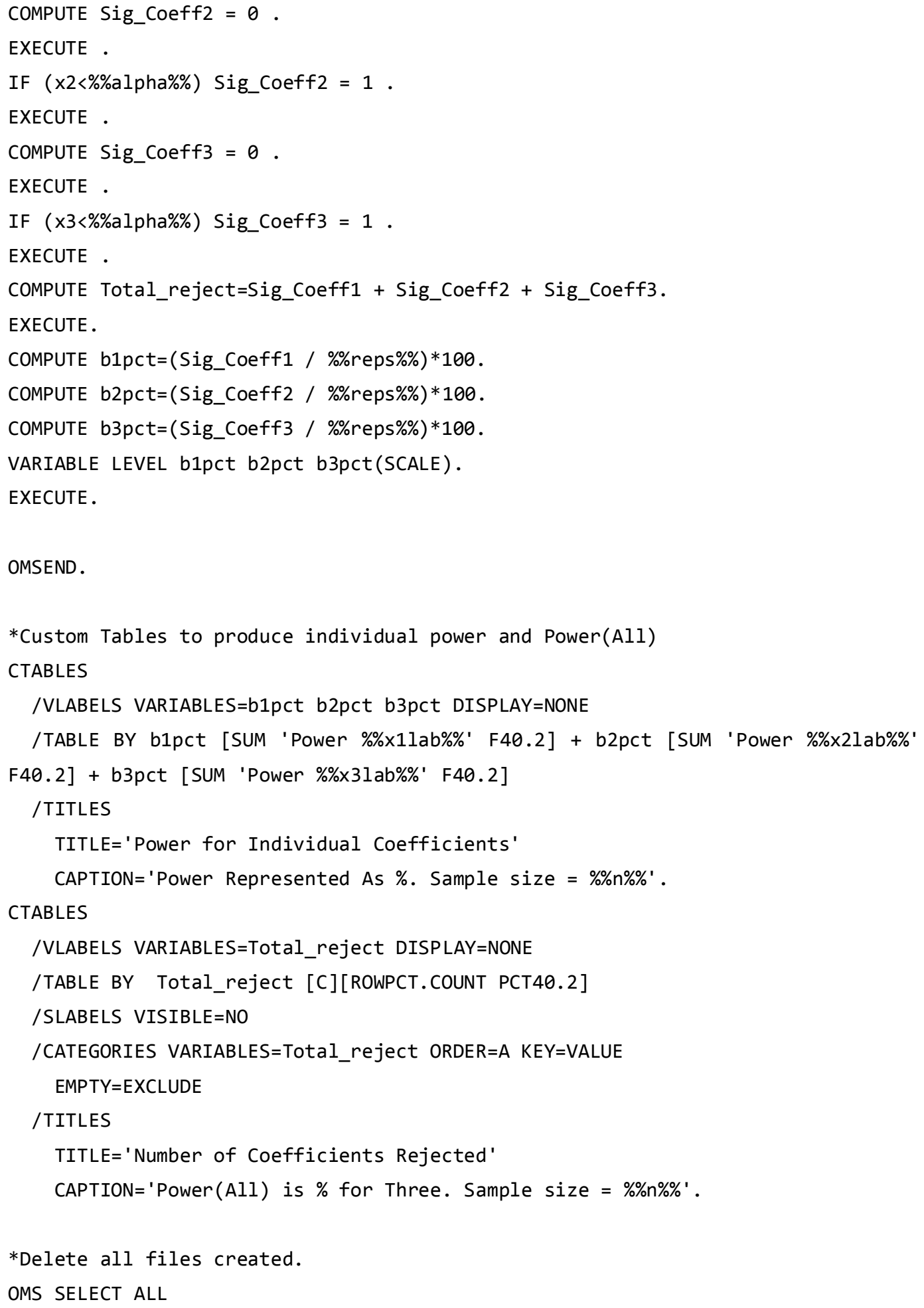




\section{CHRISTOPHER ABERSON}

/DESTINATION VIEWER=NO.

New File.

DATASET CLOSE boot1.

Erase File= '\%dir\% \corrmat.sav' .

Erase $\mathrm{File}={ }^{\prime} \%$ dir\% $\$ giant_datafile.sav' .

Erase $\mathrm{File}=$ '\%dir\% \boot1.sav' .

Omsend. 